\title{
Beyond Traffic Depiction: Conformally Integrating the Conflict Space to Support Level 3 Situation Awareness
}

\author{
Jochum Tadema ${ }^{\text {la }}$, Erik Theunissen ${ }^{\mathrm{a}, \mathrm{b}}$, Kevin M. Kirk $^{\mathrm{c}}$ \\ ${ }^{a}$ Netherlands Defence Academy, Het Nieuwe Diep 8, 1781 AC, Den Helder, The Netherlands; \\ ${ }^{\mathrm{b}}$ Delft University of Technology, Mekelweg 4, 2628 CD, Delft, The Netherlands; \\ ${ }^{\mathrm{c}}$ General Atomics Aeronautical Systems, 14200 Kirkham Way, Poway, CA 92064.
}

\begin{abstract}
The research described in this paper explores the addition of conformally integrated traffic probes into an egocentric Synthetic Vision (SV) Primary Flight Display (PFD). The underlying thought is that, although the traffic that is predicted to cause a future loss of separation may not lie within the field of view of the display, the location where the loss of separation is predicted to occur always will. Hence, rather than focusing on the depiction of traffic, which contributes to level 2 Situation Awareness (SA), the concept pursues spatially integrated depiction of the airspace where a loss of separation is predicted. This provides readily actionable conflict information, relieving pilots from the traffic position and conflict estimation task and contributing to level $3 \mathrm{SA}$. The paper describes the integration of the data from the traffic probe into an SV PFD. The advantages of the concept will be illustrated using several traffic conflict scenarios, including an overtaking scenario involving unmanned aircraft. Given that unmanned aircraft may be markedly slower than manned aircraft which operate within the same airspace, a spatially integrated depiction of airspace where a future loss of separation is predicted, can help to preserve safety in classes of airspace that accommodate both manned and unmanned aircraft. Additionally, examples are provided illustrating how traffic probes can support pilots in monitoring the conformance of traffic to the priority rules of 14 CFR 91.113.
\end{abstract}

Keywords: Synthetic Vision, conflict probing, conformal integration, traffic awareness.

\section{INTRODUCTION}

Synthetic Vision (SV) systems show a computer generated image of the environment with a quality that is independent of actual visibility conditions. The premise is that safety will increase because the timely detection of hazards is no longer impaired by (poor) visibility. Ample research data is available that supports this claim for hazards that are static, such as terrain and obstacles. However, for traffic this is not the case. The depiction of traffic to aid the pilot in detecting a potential future loss of separation has been the subject of a range of studies [1-5]. Several studies focused on questions such as dimensionality of the display, intruder symbology and presence of elements such as speed vectors. These studies implicitly make the assumption that the extrapolation of the current state to the future is performed by the pilot. Early studies concerning collisions of radar equipped vessels already found that 'Ships' officers find it difficult to properly understand the relative motion problem' [6]. Similar observations have been made regarding TCAS traffic display misinterpretation [7].

In Ref. [5], the depiction of traffic on a SV Primary Flight Display (PFD) and Navigation Display (ND) is proposed. With the traffic geometry shown in the example used in that paper, the pilot indeed sees the other traffic on the PFD. However, many conflict geometries are possible that will lead to a loss of separation and a collision hazard without the intruding aircraft ever coming within the field of view used to render the synthetic environment of a typical egocentric SV PFD. Research has addressed this issue by designing exocentric views for the depiction of traffic, providing a perspective all-around view relative to ownship. Ref. [2] discusses the evaluation of an Integrated Hazard Display (IHD), to be used in conjunction with the PFD, stating that 'The IHD must of necessity be presented from a different frame of reference than is typically used with the ego-referenced or "immersed" SVS primary flight display, because this frame of reference does not readily present information beside and behind the aircraft, critical for traffic awareness'. The study compares a 2D coplanar, 3D exocentric, and split-screen display, finding that traffic position estimation performance is

${ }^{1}$ j.tadema@nlda.nl; phone: +31-223-657545; fax: +31-223-657319; http://www.uav.nl

Enhanced and Synthetic Vision 2010, edited by Jeff J. Güell, Kenneth L. Bernier, Proc. of SPIE Vol. 7689, 768902 - @ 2010 SPIE · CCC code: 0277-786X/10/\$18 - doi: 10.1117/12.852392 
best supported by the 2D coplanar display, as the other displays suffer from perceptual ambiguity (3D) and inappropriate attention allocation (split-screen).

Rather than using a different viewpoint, the research described in this paper explores the addition of conformally integrated traffic probes [8] into an egocentric SV PFD. The underlying thought is that, although the traffic that is predicted to cause a future loss of separation may not lie within the field of view, the location where the loss of separation is predicted to occur always will. Hence, rather than focusing on the depiction of traffic, which contributes to level 2 Situation Awareness ${ }^{2}$ (SA), the concept pursues a spatially integrated depiction of the airspace where a future loss of separation is predicted. This provides readily actionable conflict information, relieving pilots from the traffic position and conflict estimation task and contributing to level 3 SA. Depending upon the concept of operation, the traffic probe can be configured using either a pilot-selectable Assured Normal Separation Distance (ANSD) [10] or a Self Separation Threshold (SST) [11].

The paper will start by briefly revisiting concept of conflict probing, followed by a discussion on the integration of the data from the traffic probe into a SV PFD, including potential cluttering issues. Where applicable within the scope of this paper, references will be made to criteria for performance-based requirements of future separation assurance and collision avoidance systems, given in Ref. [12]. The advantages of the concept will be illustrated using several traffic conflict scenarios, including an overtaking scenario involving unmanned aircraft. Given that unmanned aircraft may be markedly slower than manned aircraft which operate within the same airspace, a spatially integrated depiction of airspace where a future loss of separation is predicted, can help to preserve safety in classes of airspace that accommodate both manned and unmanned aircraft. Additionally, examples are provided illustrating how traffic probes can support pilots in monitoring the conformance of traffic to the priority rules of 14 CFR 91.113 [13]. Finally, a scenario addressing solution stability requirements and multivector resolution strategies is provided.

\section{CONFLICT PROBING AND INTEGRATED CONFLICT AVOIDANCE}

Conflict probing consists of a prediction of the future separation between ownship and hazards for a set of ownship velocity vectors -i.e., possible combinations of Track, Flight Path Angle (FPA) and Speed- up to a predefined prediction horizon or look-ahead time. Using predefined separation criteria, e.g., thresholds used to define a loss of separation or a collision hazard, the probing data indicates which ownship velocity vectors will lead to a future conflict and what the corresponding time to loss of separation is.

To illustrate the concept of probing, Fig. 1 shows a top view of an example conflict geometry resulting from the presence of converging traffic. Vehicle A (center) represents ownship, vehicle $\mathrm{B}$ is the intruder aircraft; the dashed lines represent the current Tracks. Initial Bearing of the intruder is 290, initial Range is $5 \mathrm{NM}$; the intruder's Track is 050 , flying level at a Speed of 250 kts. Both airplanes are at the same Flight Level. The depicted conflict track band and conflict probe result from probing for a range of variations of the ownship Track angle $\left(\Psi_{\mathrm{A}}\right)$, for the current FPA and Speed. The separation criteria (yellow) used in this example are $1 \mathrm{NM}$ lateral and $1 \mathrm{kft}$ vertical. The collision hazard criteria (red) used in this example are $0.25 \mathrm{NM}$ lateral and $500 \mathrm{ft}$ vertical. The corresponding time to loss of separation (T2LOS) for a range of variations of all three velocity vector dimensions is depicted as a color coded object in Fig. 2a. The depicted 'volume' represents the conflict space in the velocity vector

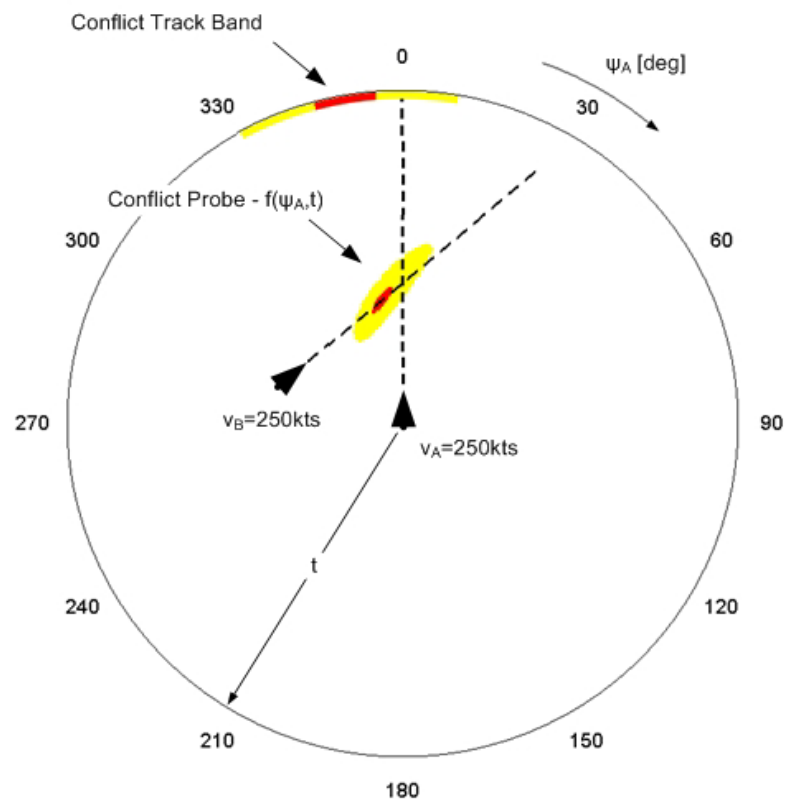

Figure 1. Top view of an example conflict geometry and Conflict Probing in the Track $\left(\Psi_{\mathrm{A}}\right)$ domain. The conflict probe indicates the ownship tracks that will result in a violation of the separation (yellow) or collision hazard criteria (red).

\footnotetext{
${ }^{2}$ Situation Awareness is defined as: the perception of the elements in the environment within a volume of time and space (Level 1 $\mathrm{SA}$ ), the comprehension of their meaning (Level $2 \mathrm{SA}$ ) and the projection of their status in the near future (Level 3 SA) [9].
} 
domain, providing a 'translation' of the relative motion problem, to a set of velocity vectors that should be avoided. All combinations of ownship Track $\left(\Psi_{\mathrm{A}}\right)$, FPA $\left(\gamma_{\mathrm{A}}\right)$ and Speed $\left(\mathrm{v}_{\mathrm{A}}\right)$ that lie outside of this volume represent possible conflict prevention/resolution maneuvers. Hence, information about the trade-offs and interdependencies of changes of the velocity vector components can be readily obtained from the structure, supporting multi-dimensional conflict resolution. Figs. 2b-d show 'slices' of the 3D data structure for the original speed $\left(\mathrm{v}_{0}\right)$, FPA $\left(\gamma_{0}\right)$ and Track $\left(\Psi_{0}\right)$. E.g., Fig. $2 \mathrm{~b}$ represents the conflict space in the Track-FPA domain. This data can be conformally integrated into a HUD or SV PFD, providing a readily actionable representation of the maneuver space that is available with respect to traffic.

As probing is performed in real-time, the conflict space (i.e., its 'position', shape and T2LOS content) is continuously updated while the situation develops. Should intruder aircraft maneuver, this will be reflected by corresponding changes of the conflict space. By storing the probing data as presented here, as T2LOS records for the variations of the ownship velocity vector, the temporal characteristics of potential conflicts are retained, allowing prioritization and timing of avoidance maneuvers.

Conflict probing is currently being considered as a means to integrate the alerts from multiple hazard detection systems in the context of research performed for the NASA IIFD program [14]. In [8] it is discussed how conflict probing can provide a common framework for the computation of coordinated conflict avoidance maneuvers that include integration of multiple types of hazards and constraints such as vehicle performance and right-of-way rules. To illustrate this, Fig. 3 depicts the elements and information flow of a conflict avoidance concept that uses conflict probing as a framework for integrating data.
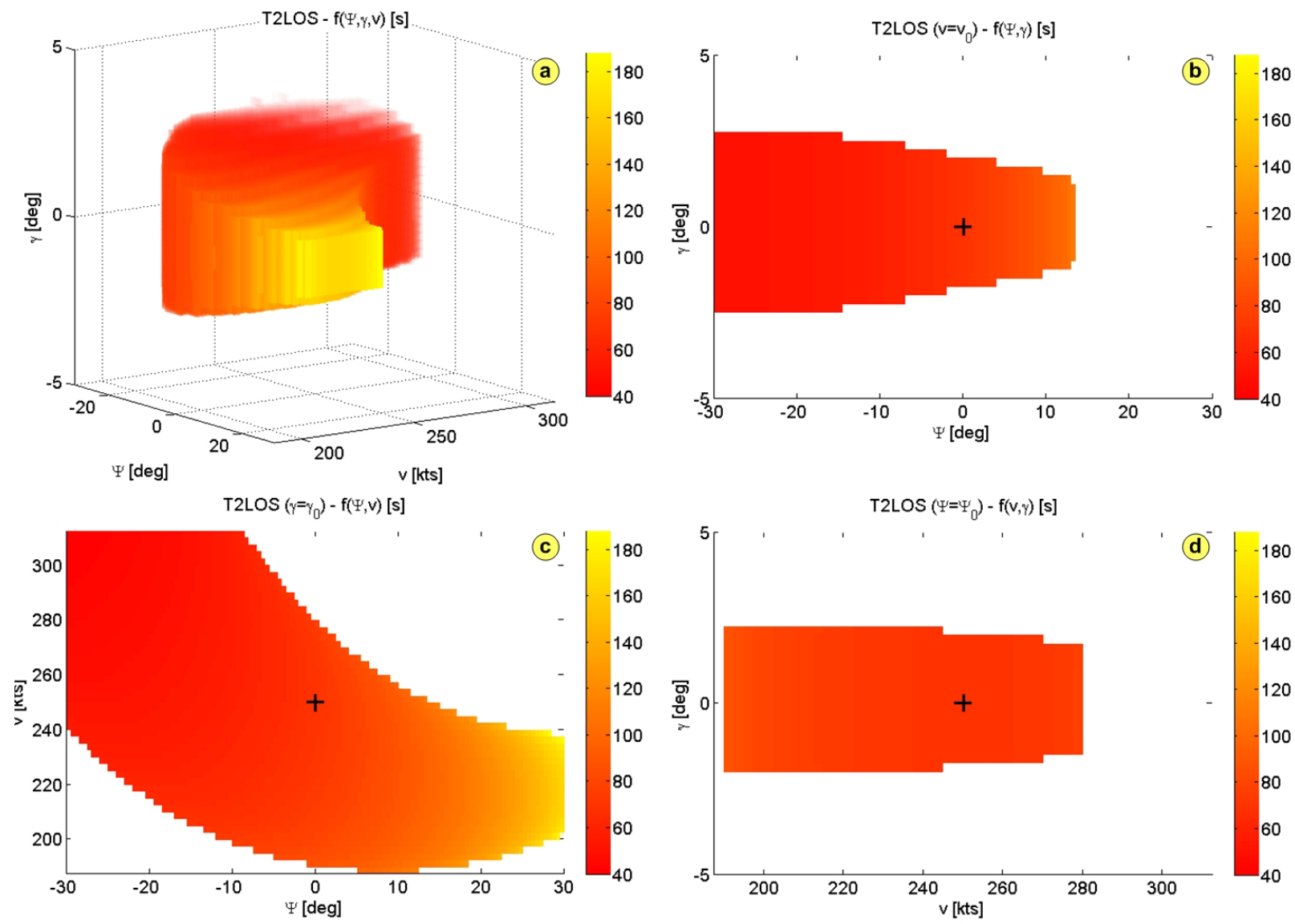

Figure 2. Example conflict space presented in the three domains constituting the flight path vector. 


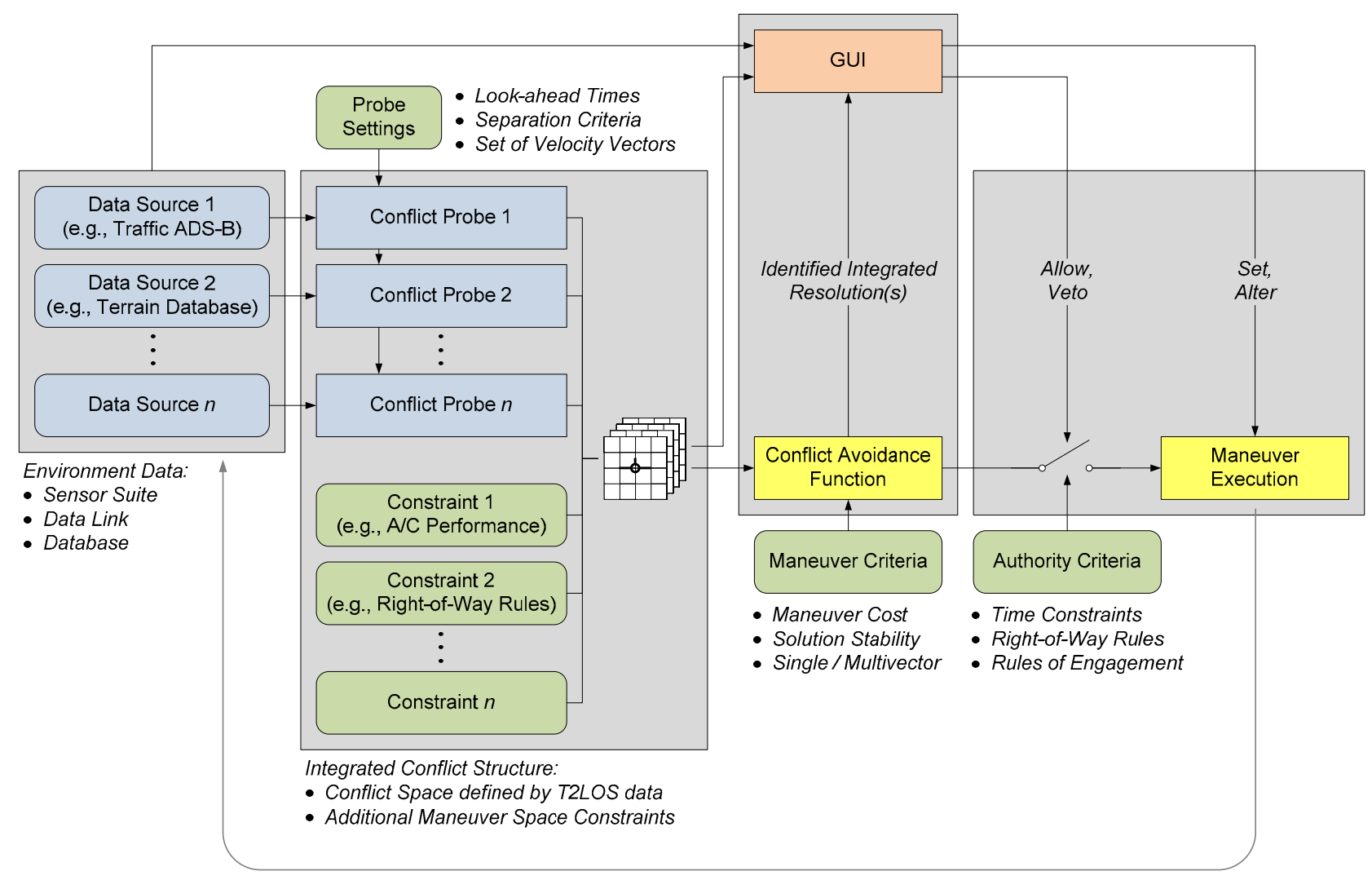

Figure 3. Elements and information flow of an integrated conflict avoidance concept based on probing.

The integrated conflict structure contains the conflict space, defined by the output of the conflict probes ${ }^{3}$, and additional constraints to the maneuver space. Each cell in the structure represents a specific combination of Track, FPA and Speed; the value of the cell indicates whether that velocity vector leads to a conflict or whether it has to be excluded for any other reason. E.g., aircraft performance characteristics pose a fundamental constraint on the solution space. Procedural constraints such as the right-of-way rules [13] can be regarded as a set of situation dependent 'no-go' directions, and can be implemented as such, as one of the structure's layers.

In the approach presented in [15], performance limitations are specified as constraints for each of the conflict resolution vectors that are computed. The available 3D space for conflict resolution can be maximized by combining vertical and lateral maneuvers, and utilizing the ability to convert the available speed margin relative to $\mathrm{v}_{\min }$ or $\mathrm{v}_{\max }$ (kinetic energy) into altitude (potential energy). For humans it is almost impossible to maximize the maneuvering performance in this way without violating one or more maneuvering constraints such as angle of attack, stall speed, load factor and bank angle. In [16] it is illustrated how information about the maximum safe maneuvering authority is integrated into the conflict prevention/resolution function of a probe using integrated control authority allocation and envelope protection functionality.

\section{DISPLAY INTEGRATION}

When properly integrated into a Graphical User Interface (GUI), the data contained in the integrated conflict structure provides the ability of the pilot to assess the situation; it supports level 3 SA by enabling pilots to anticipate the results of changes to the current velocity vector. Additionally, a conflict avoidance function can be used to identify one or more possible avoidance maneuvers from the structure by applying criteria such as solution stability and maneuver cost [17]. Based on the Level Of Authority (LOA) for which the system is designed to operate, these identified avoidance maneuvers can either serve to support the pilot's decisions, or can be executed automatically. This section addresses the

\footnotetext{
${ }^{3}$ The 3D data structure depicted in Fig. 2a is one of the multiple layers, or pages, in the integrated conflict structure.
} 
integration of probing data into the plan-view Cockpit Display of Traffic Information (CDTI) and the SV PFD of our research simulator $[18,19]$. Additionally, potential cluttering issues related to the addition of traffic probes are explored.

\subsection{Probing data in a plan-view CDTI}

Track probe data such as shown in Fig.1 can be conformally mapped onto a spatial reference frame for integration into a plan-view CDTI. This is illustrated by Fig. 4, showing an example scenario involving two other aircraft. Traffic is depicted by directional symbols (with distinctions for 'other' and proximity traffic) and track labels indicating the speed [kts] and the altitude difference [Flight Levels]. The separation criteria (yellow), that are used as probe settings, in this example are $1 \mathrm{NM}$ lateral and $1 \mathrm{kft}$ vertical. The collision hazard criteria (red) used here are $500 \mathrm{ft}$ lateral and $500 \mathrm{ft}$ vertical. These probe settings will be used for the remainder of this paper. The dashed lines represent the horizontal field of view of the SVS PFD. These lines and the numbers and letters used to indicate the traffic and probes are not part of the display and are included here for illustrational purposes.

Probe area A indicates where a loss of separation is predicted to occur with aircraft 1 . Although the current path is conflict free, the pilot can instantly see that maneuvering to the left would result in a future Loss of Separation with oncoming aircraft 1 . The display represents a plan view intersection of the conflict probes along ownship's current FPA. Therefore, conflict probes belonging to aircraft with a predicted vertical separation exceeding the separation criteria (here aircraft 2, being $3 \mathrm{kft}$ below ownship's current path) are not depicted.

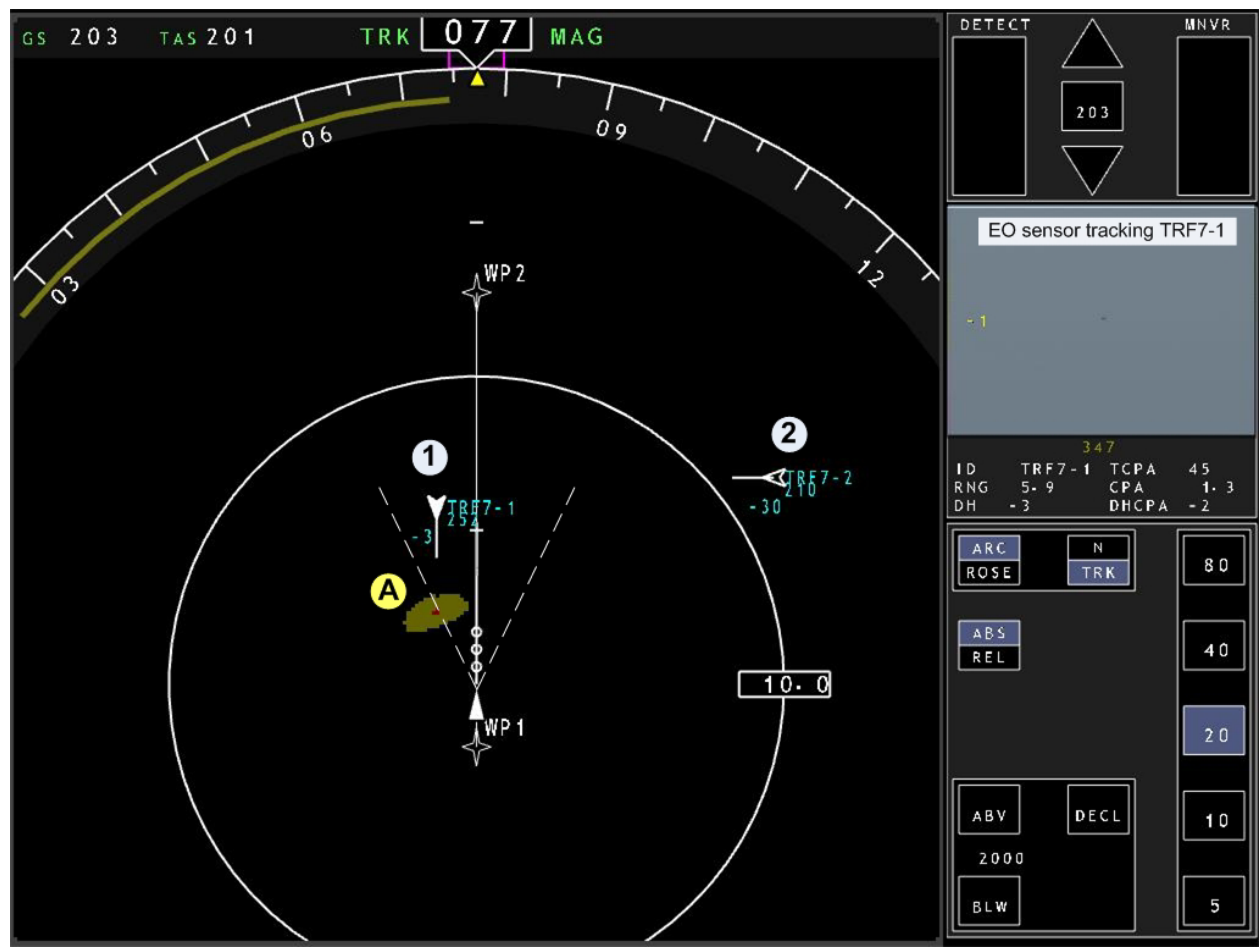

Figure 4. Plan-view conflict probe display, depicting an example scenario with two other aircraft.

\subsection{Probing data in an egocentric perspective SV PFD}

Figure 5 shows the same situation, depicted on a SV PFD with integrated Track-FPA probe (data such as illustrated in Fig. 2b). The probe data is conformally integrated as color-coded objects, representing the velocity vector directions that will result in a future loss of separation. The color of the object represents the time to loss of separation. The SV PFD provides an integrated depiction of both the horizontal and vertical components of the conflicts. In addition to the conflict probes intersected by the current FPA that are depicted on the plan-view CDTI, such as probe A, this also allows for the depiction of conflict probes belonging to aircraft with a predicted vertical separation exceeding the separation criteria, in this example aircraft 2 causing conflict probe B. The color difference between probe A and B indicates that conflict A has a shorter time to loss of separation than conflict B. Aircraft 1 is within the field of view, so its position can be conformally integrated in the display, as was suggested by e.g., Ref. [5]. Although aircraft 2 is outside the PFD field 
of view, its conflict probe B is not. Consequently, with the probes in the SVS PFD, the pilot is provided with full awareness of the directional constraints on the flight path vector imposed by both aircraft, supporting the assessment of the situation, independent of traffic depiction.

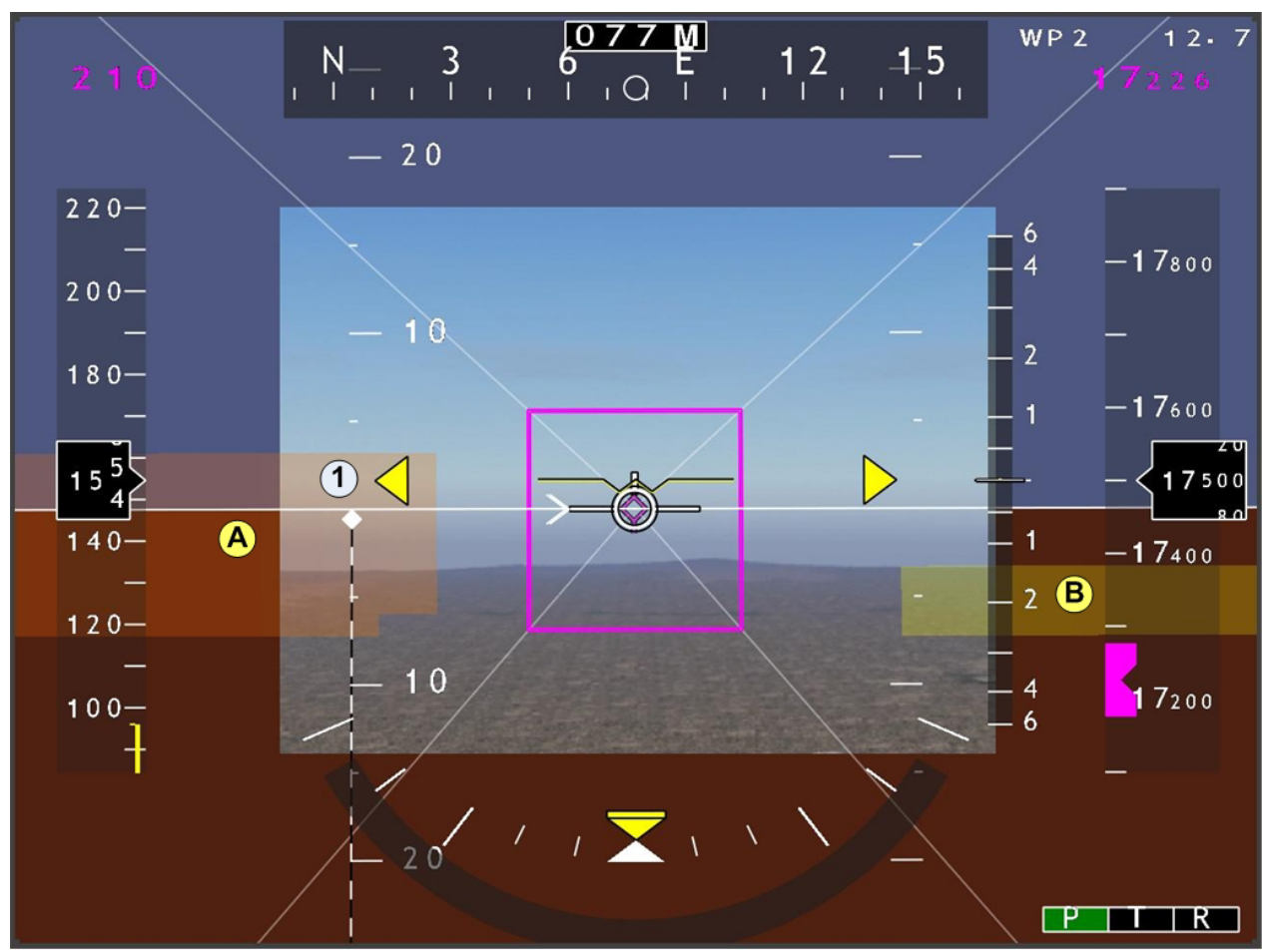

Figure 5. SV PFD with integrated Track-FPA probe, depicting the situation as displayed in Fig 4, from an egocentric perspective viewpoint.

\subsection{Dense traffic situations and potential clutter issues}

Adding additional symbology and objects to existing display formats can potentially result in clutter issues. The probing concept is able to handle multiple intruders. When there is more than one intruder, the probing algorithm will generate additional areas where a loss of separation is predicted. The amount of the areas that will be displayed is a function of the look-ahead time (i.e., the prediction horizon); the size of the areas is a function of the separation criteria and data uncertainty. For the same traffic density, the amount of areas will increase with an increasing number of aircraft, but the location of these areas will also be further away from ownship. Because the effect of uncertainty in track and speed increases with an increase in look-ahead time, also the size of the area will increase.

To explore the potential cluttering caused by the integration of traffic probes, consider Fig. 6, showing a high density traffic situation involving 6 other aircraft. Aircraft 5 and 6 do not cause any potential separation conflicts. Oncoming aircraft 1 and 3 result in conflict probes A and C on the CDTI (Fig. 6a). Aircraft 1, 2 and 4 cause conflict probes A, B and D on the SV PFD (Fig. 6b). Conflict probe $C$ is outside of the PFD field of view; so are aircraft 4 and 5 . Aircraft 3 is within the PFD field of view, but beyond the threshold chosen for traffic depiction.

This example illustrates that additional traffic increases the number of depicted probes. However, in contrast to the depicted traffic symbology, traffic probes that are based on an appropriate prediction horizon, represent readily actionable information that is relevant for near-term separation assurance. In fact, based on Fig. 6, one could even argue that the traffic symbology itself, and especially the track labels provided on the CDTI, might be more prone to cluttering than the addition of the conflict probes. 


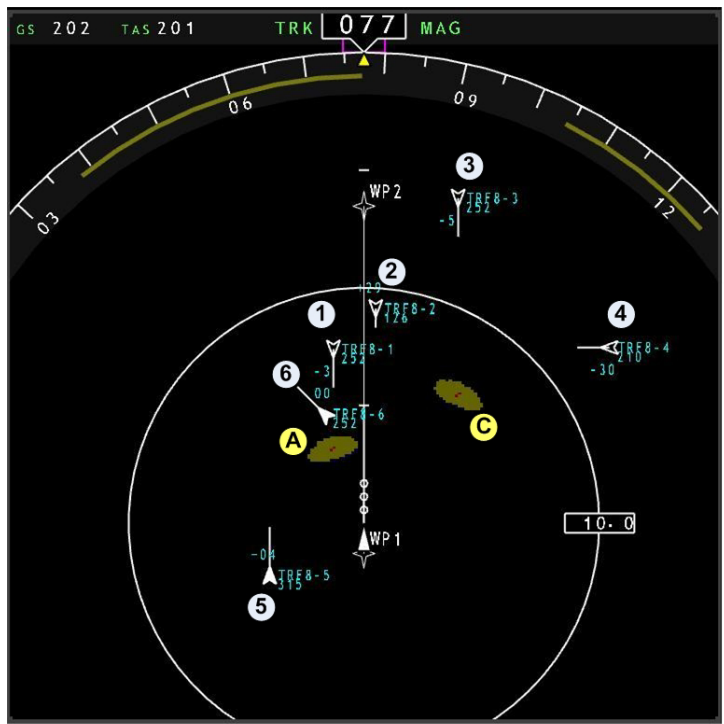

(a)

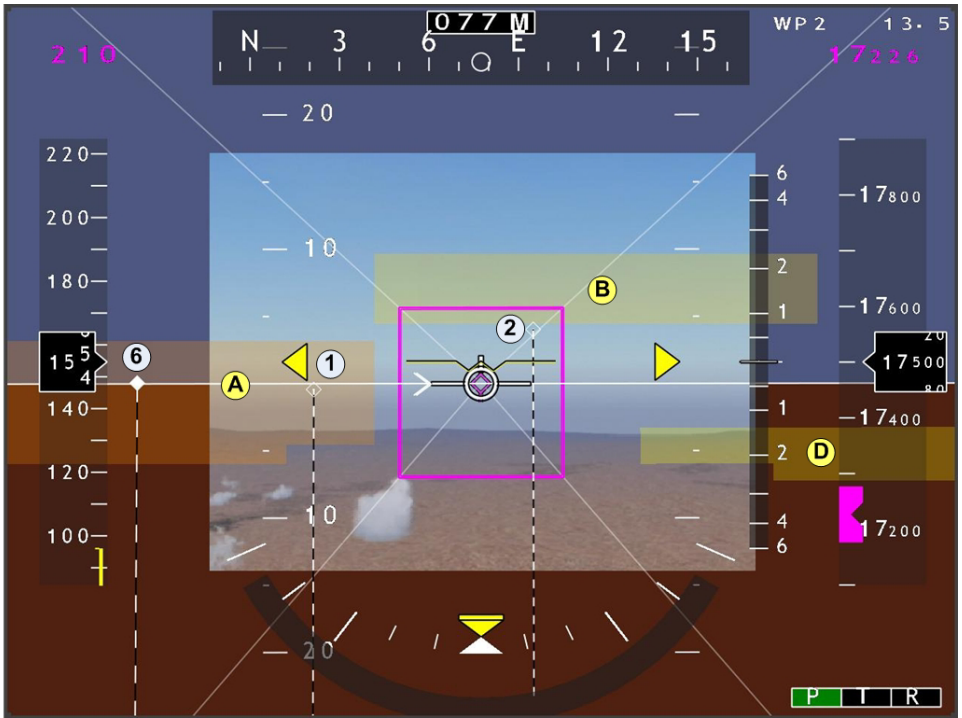

(b)

Figure 6. Exploring potential cluttering issues in high density traffic situations.

\section{EXAMPLE SCENARIOS}

Following the discussion on the general principles of conflict probing and the integration of probing data into the CDTI and SV PFD, this section illustrates the advantages of the concept using several traffic conflict scenarios, including overtaking scenarios involving unmanned aircraft. Given that unmanned aircraft may be markedly slower than manned aircraft which operate within the same airspace, a spatially integrated depiction of airspace where a future loss of separation is predicted, can help to preserve safety in classes of airspace that accommodate both manned and unmanned aircraft. The overtaking scenarios illustrate how different types of resolution maneuvers are supported, by showing both lateral and vertical resolution strategies. Additionally, examples are provided illustrating how traffic probes can support pilots in monitoring the conformance of traffic to the right of way priority rules of 14 CFR 91.113. Finally, a scenario addressing solution stability requirements and multivector resolution strategies is provided.

\subsection{Overtaking scenario - Lateral maneuver}

In this first scenario, ownship represents a relatively slow unmanned aircraft, that is being overtaken by faster vehicle in a situation involving multiple aircraft. While the situation develops, the overtaker blunders into the planned path of the unmanned aircraft, requiring an evasive maneuver to maintain separation. It will be shown that although the actual intruder never comes within the field of view of the SV PFD, with the addition of traffic probes, ownship's pilot is fully aware of the imminent threat and able to successfully avoid the separation conflict, without creating a new conflict with the other aircraft or terrain.

Figure 7 shows the initial situation. The plan-view CDTI shows four other aircraft relative to ownship. The current path is conflict free, but maneuvering too much to the left or right would result in a future loss of separation with oncoming aircraft 3 and 4, as indicated by the conflict probes $\mathrm{C}$ and $\mathrm{D}$. The predicted vertical separation of aircraft 1 and 2 exceeds the required separation and therefore the probes belonging to those aircraft are not depicted on the CDTI. The SV PFD does show these probes A (aircraft 1) and B (aircraft 2), in addition to probe C (aircraft 3). Aircraft 1, 2 and probe D are outside of the PFD field of view. Aircraft 4 is outside of the PFD's traffic depiction range. 
In Fig. 8, aircraft 1 (the overtaker) starts to descend, causing the future vertical separation to be less than $1 \mathrm{kft}$. This is reflected by the appearance of its conflict probe (A) on the CDTI. Simultaneously, aircraft 1 changed its course 4 deg to the left, causing a future separation conflict with the current flight plan. The dotted magenta line (S) represents a lateral resolution option that stays well clear of all other traffic, in case the overtaker does not recover from its mistake. The SV PFD shows how the conflict probe of the overtaker (A), has moved into the path of ownship, indicating the future separation conflict. The depicted flightplan has turned red, as an indication of this as well. The magenta diamond (S) represents the lateral resolution option corresponding to the magenta dotted line on the CDTI. Maneuver S can serve as a flight path vector (FPV) setpoint for the Automatic Flight Control System (AFCS), or can be executed manually by the pilot. Another possible resolution option revealed by integrated depiction of the Track-FPA conflict probes is climbing; as can be seen, descending might result in a new conflict, regarding the future separation with terrain.

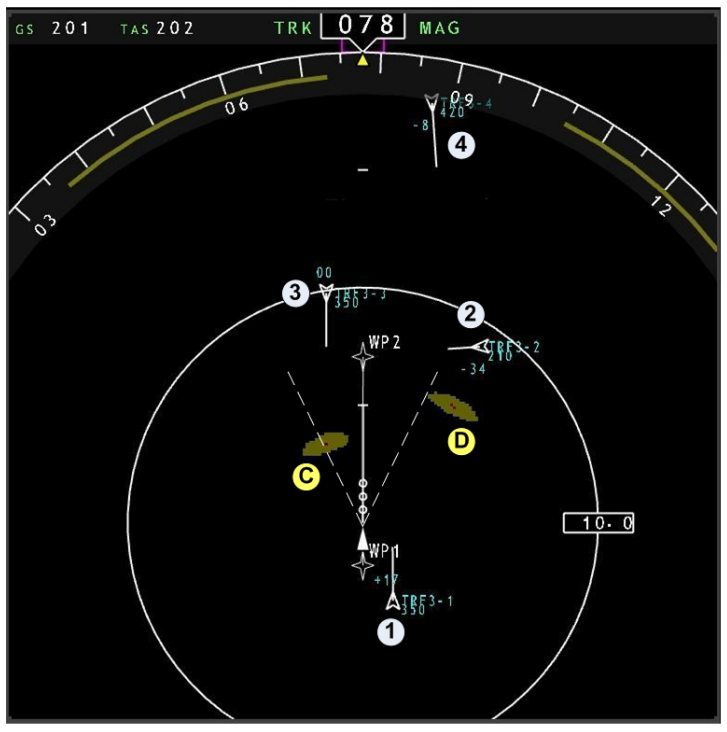

(a)

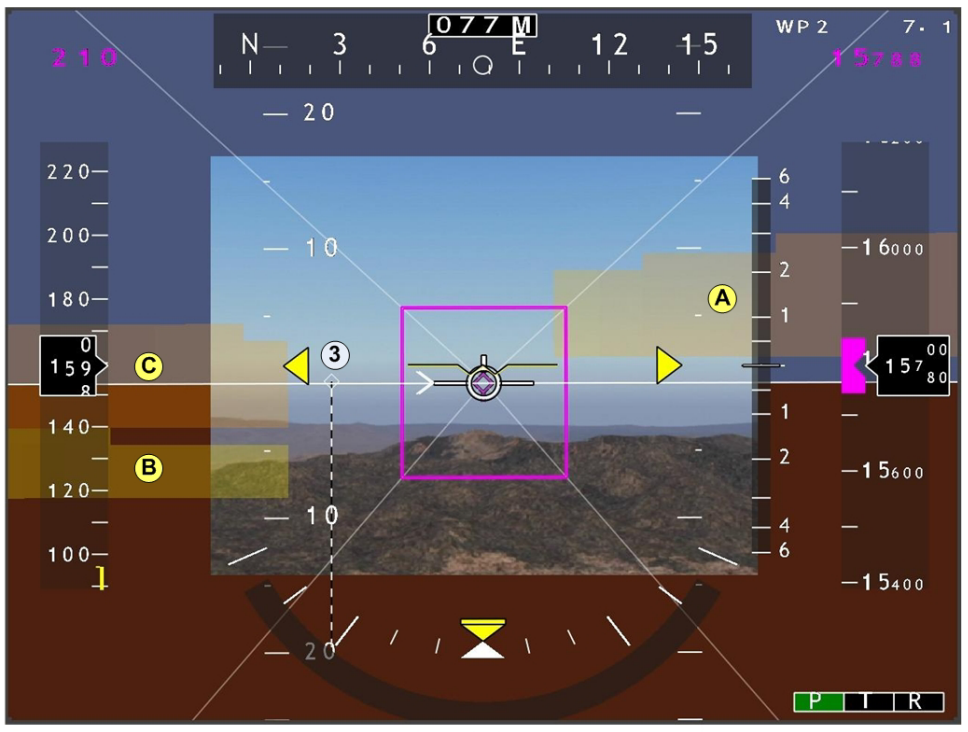

(b)

Figure 7. Initial situation in an example overtaking scenario, involving four other aircraft.

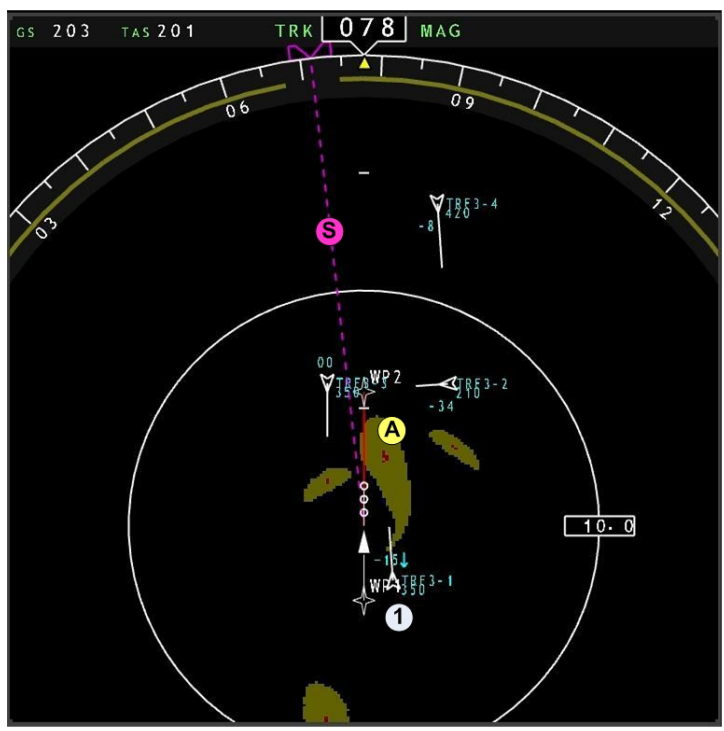

(a)

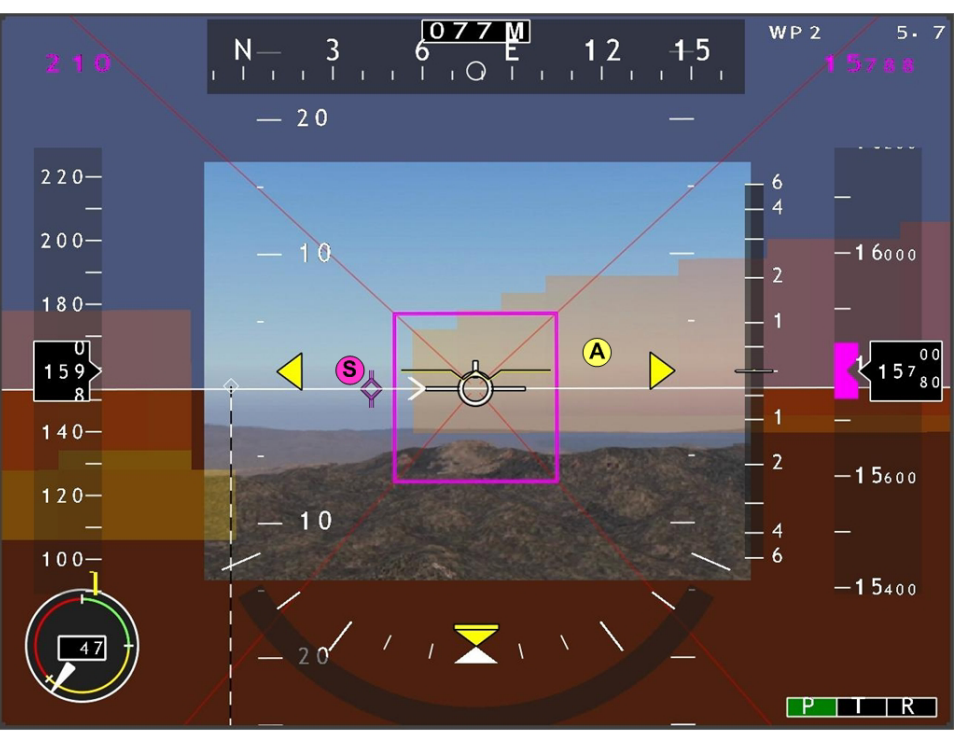

(b)

Figure 8. Aircraft 1, flying in from behind, starts to descend and changes its track to the left in such a way that a future separation conflict is created. 
Figure 9 shows how the unmanned aircraft leaves its planned path by executing the identified lateral resolution maneuver (setting maneuver S from Fig. 8 as a FPV setpoint). The probes are being updated continuously according to the developing situation. The range setting on the CDTI has been reduced to $10 \mathrm{NM}$, to provide a better overview of the maneuver space between probe $\mathrm{A}$ and $\mathrm{C}$.

Figure 10 shows the unmanned aircraft is flying alongside the planned path to stay clear of the overtaking aircraft; note that aircraft 2, passing from right to left, is now within the SV PFD field of view and depicted by the white diamond behind the altitude tape (2), although long after its probe was visible. Finally, Fig. 11 shows how overtaking aircraft 1 has passed, all potential conflicts have dissolved, and the original plan can be resumed. Note that even at this point the overtaker is still not within the PFD field of view.

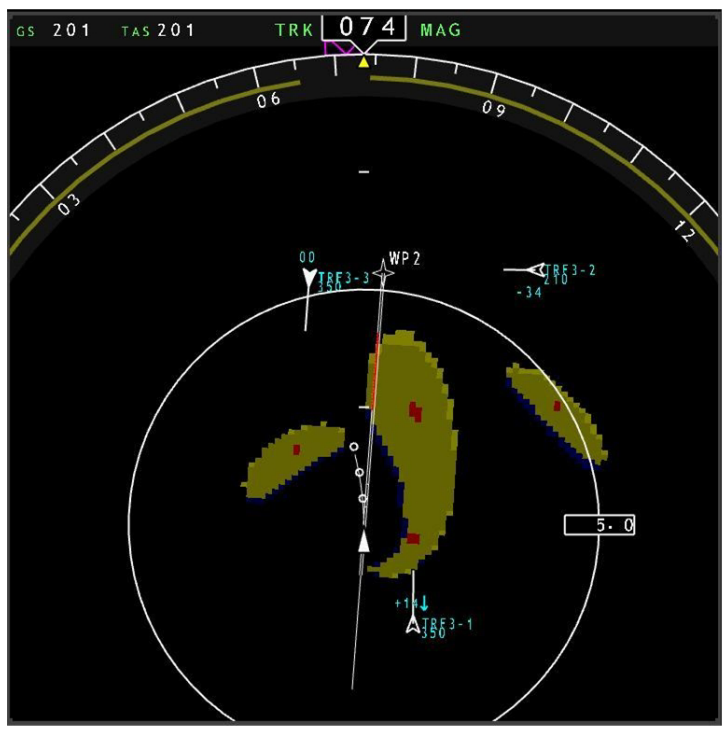

(a)

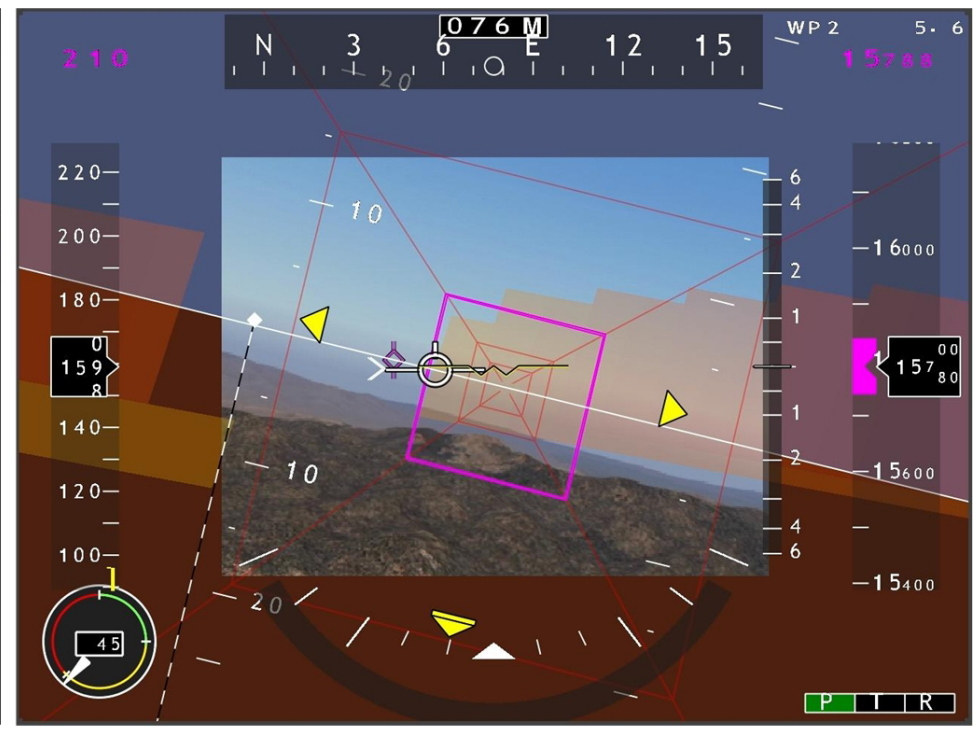

(b)

Figure 9. Executing the selected lateral resolution maneuver, while the probes are being updated according to the developing situation.

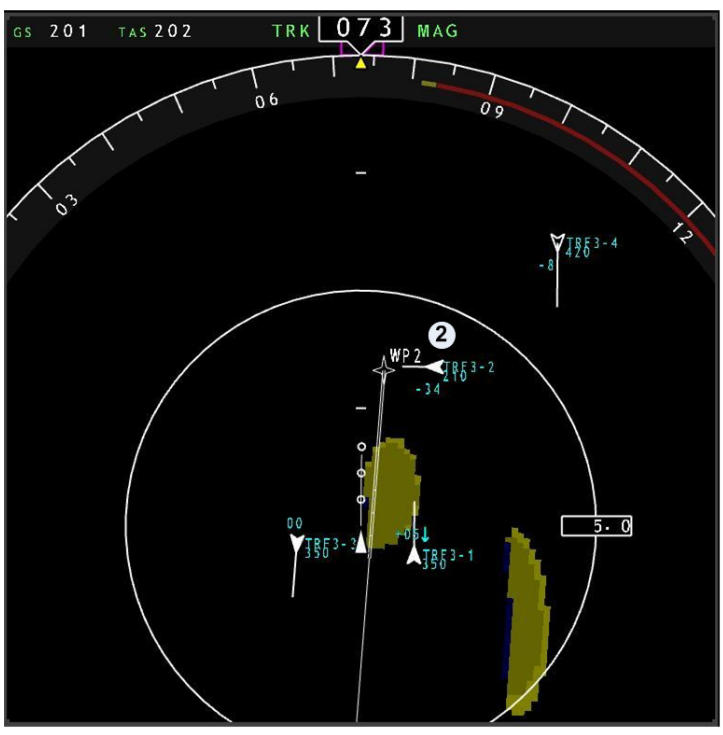

(a)

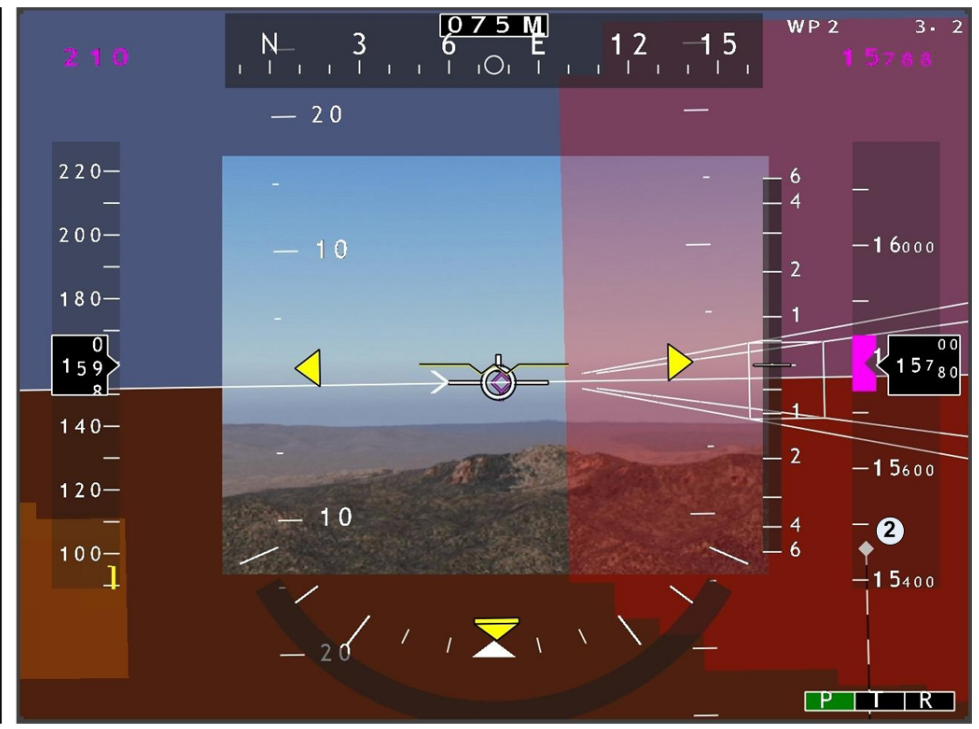

(b)

Figure 10. Flying alongside the original path to stay clear of the overtaking aircraft; note that aircraft 2, passing from right to left is now within the SV PFD field of view and depicted by the white diamond (2). 


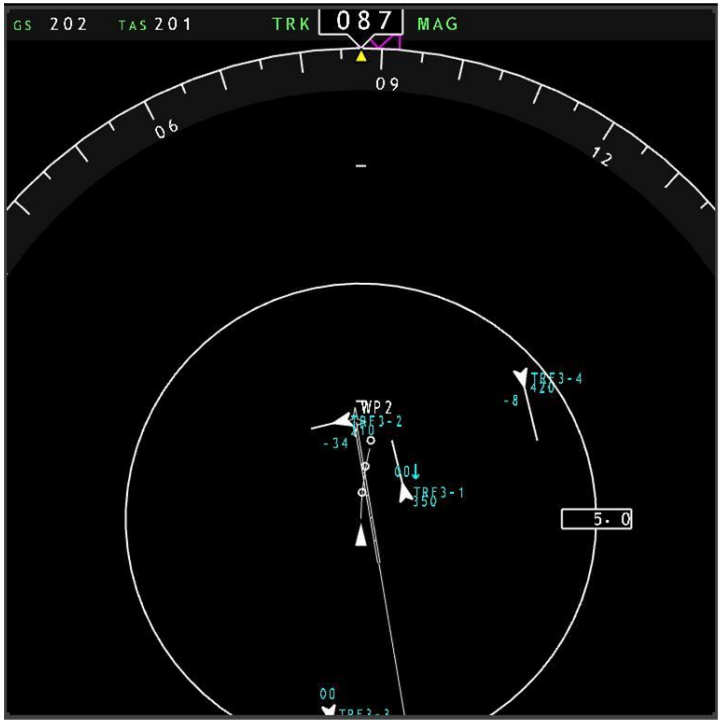

(a)

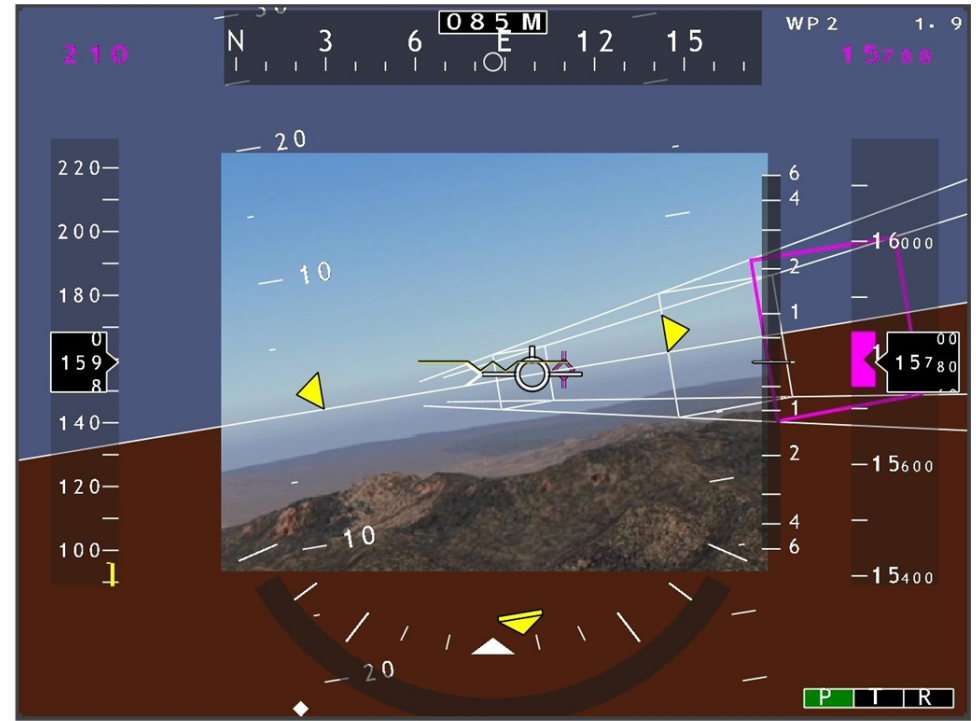

(b)

Figure 11. Overtaking aircraft 1 has passed, all potential conflicts have dissolved, and the original plan can be resumed.

\subsection{Overtaking scenario - Vertical maneuver}

The second scenario is similar to the first, except now the unmanned aircraft is on a descending flight path and the overtaking aircraft is flying level. Figure 12 shows the initial situation. The plan-view CDTI shows four other aircraft relative to ownship. The current path is conflict free, but maneuvering to the left or right would result in a future loss of separation with oncoming aircraft 3 and overtaking aircraft 1 , as indicated by the conflict probes $\mathrm{C}$ and $\mathrm{A}$. The predicted vertical separation of aircraft 2 exceeds the required separation and therefore its conflict probe is not depicted on the CDTI. The SV PFD does show probe B (aircraft 2), in addition to probe A (aircraft 1) and probe C (aircraft 3). Aircraft 1,2 and probe D are outside of the PFD field of view. Aircraft 4 is outside of the PFD's traffic depiction range.

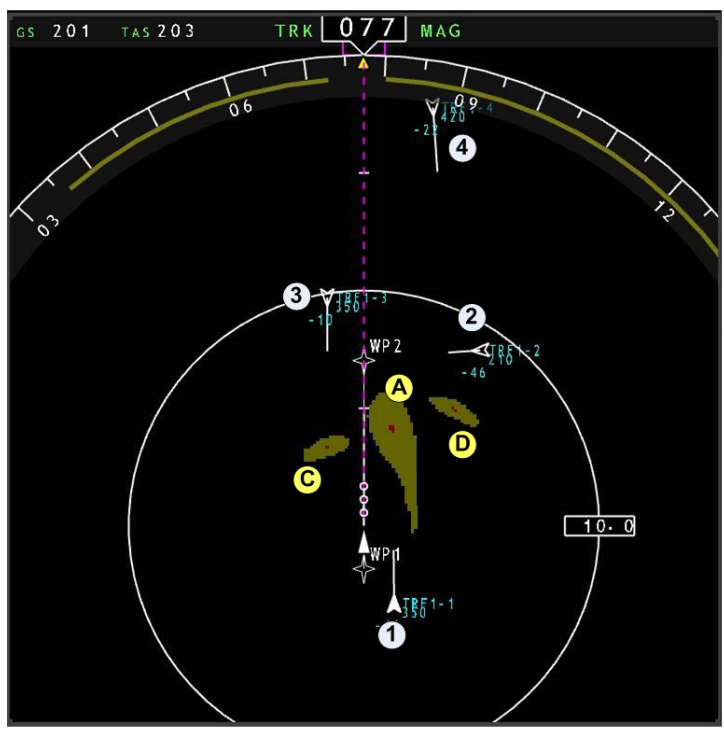

(a)

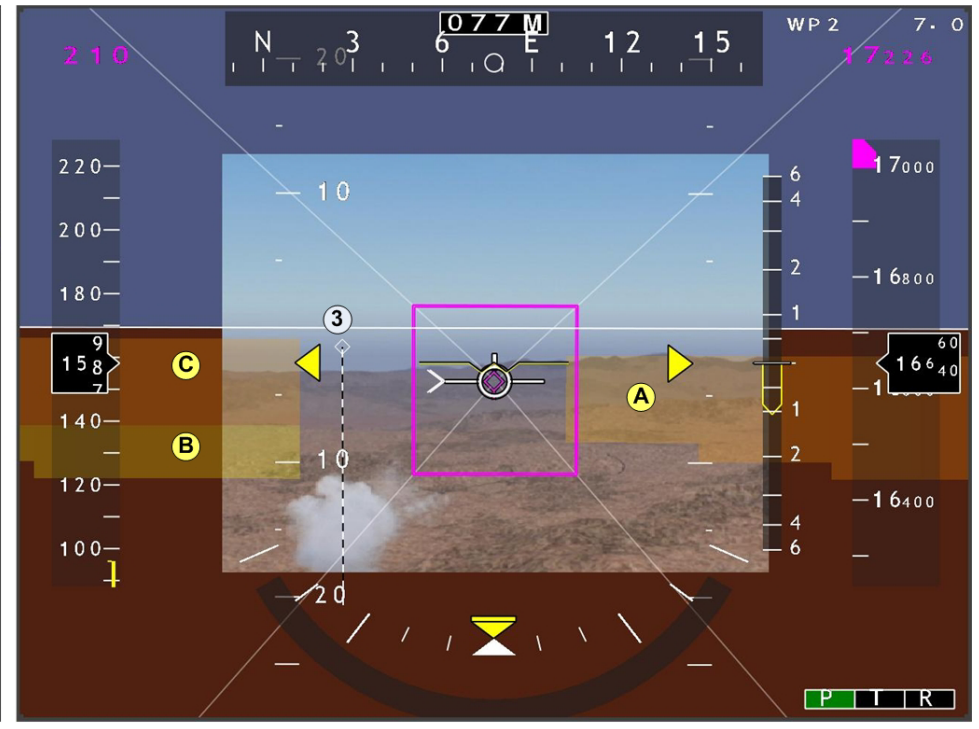

(b)

Figure 12. Initial situation in an example overtaking scenario, involving four other aircraft 
In Fig. 13, aircraft 1 (the overtaker) changes its course 4 deg to the left, causing a future separation conflict with the current flight plan. The SV PFD shows how the conflict probe of the overtaker (A), has moved into the path of ownship, indicating the future separation conflict. The depicted flightplan has turned red, as an indication of this as well. The magenta diamond (S) represents the vertical resolution option that stays well clear of all other traffic, in case the overtaker does not recover from its mistake. Figure 14 shows how the unmanned aircraft leaves its planned path by executing the identified vertical resolution maneuver. The probes are being updated continuously according to the developing situation.

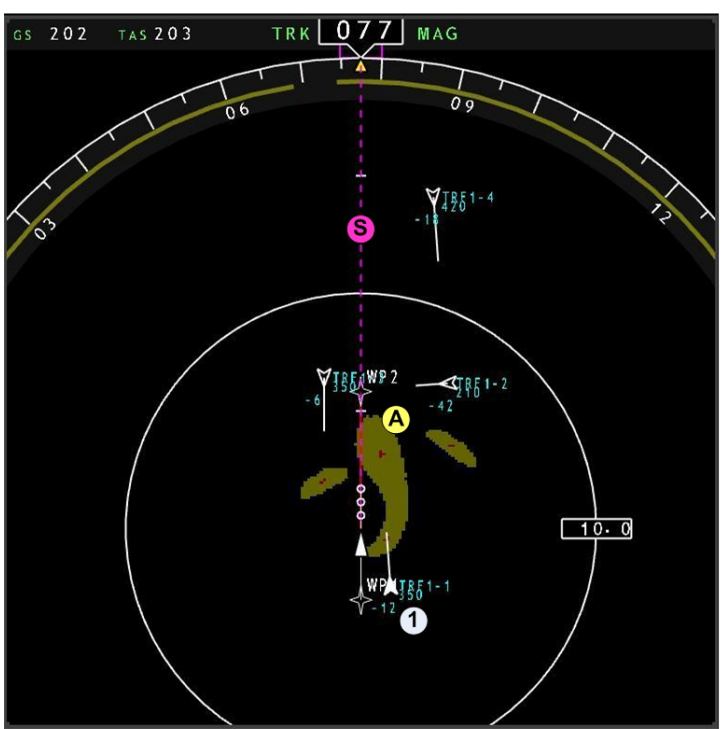

(a)

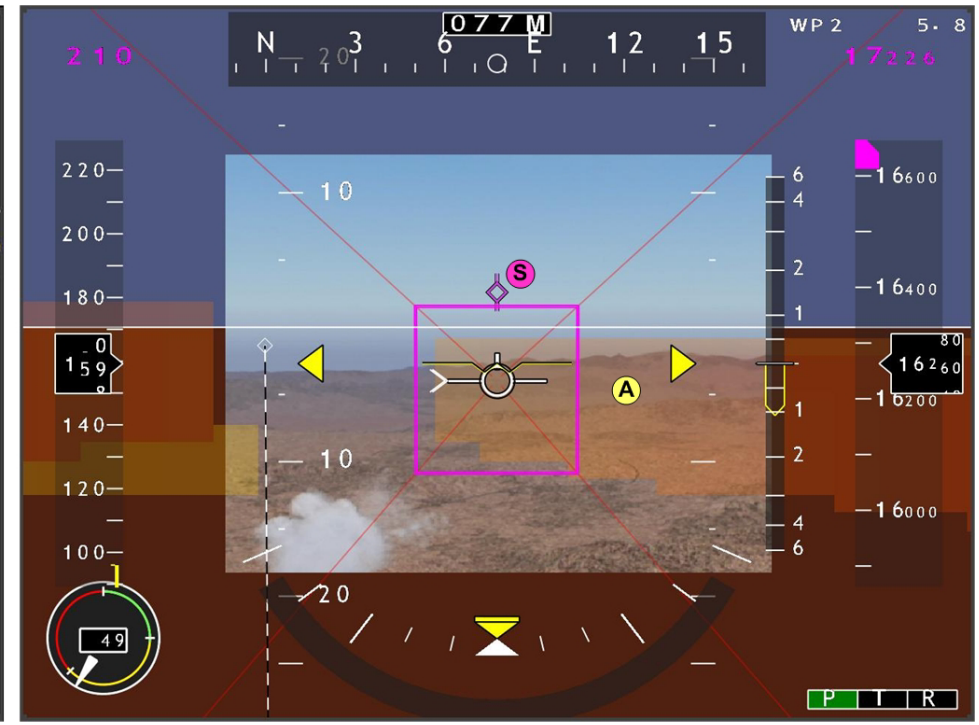

(b)

Figure 13. Aircraft 1 changes its track 4 deg to the left, causing a future separation conflict.

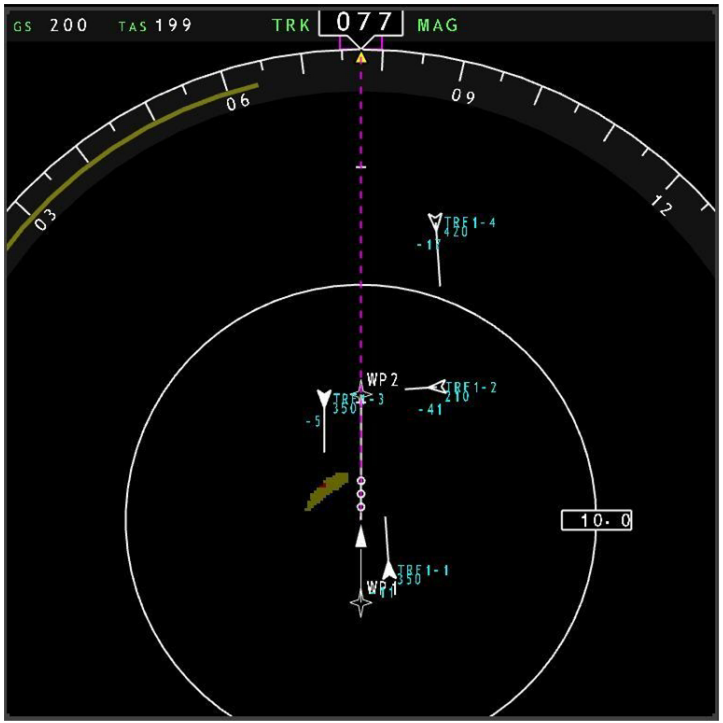

(a)

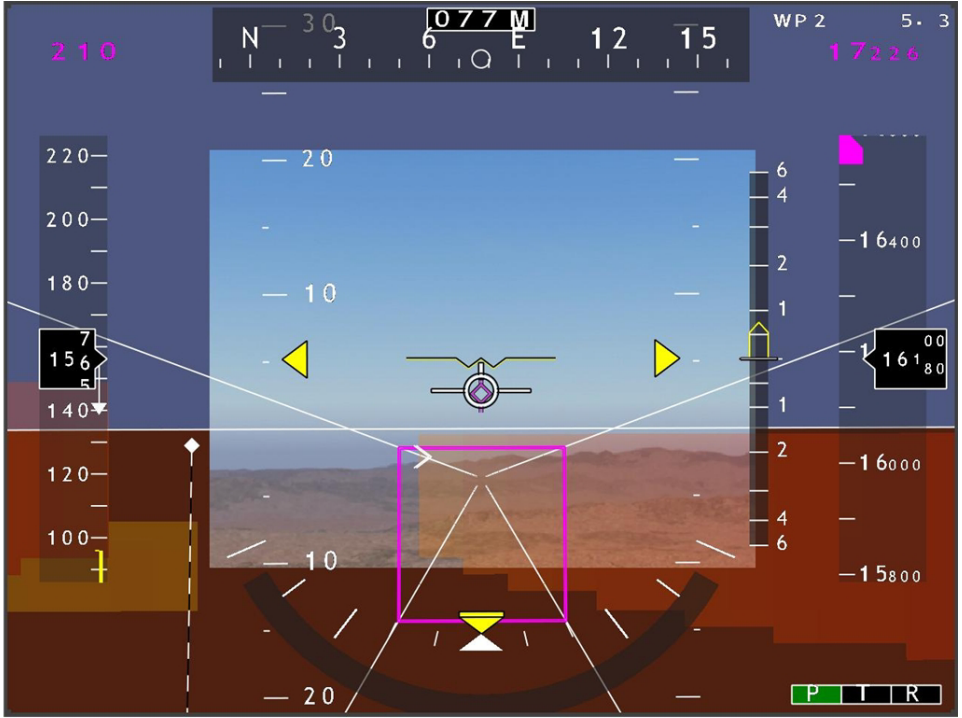

(b)

Figure 14. Executing the selected vertical resolution maneuver, while the probes are being updated according to the developing situation. 


\subsection{Monitoring traffic conformance to right-of-way priority rules}

In the previous scenarios, traffic blundering into ownship's flight path required evasive maneuvering. In the following scenario it will be illustrated how conflict probes provide the pilot with the ability to assess traffic's conformance to the right of way priority rules. Figure 15 shows the initial situation, in which aircraft 1, passing from left to right, causes a future separation conflict. Based on traffic to the priority rules of 14 CFR 91.113 [13], the intruder has to give way and change its flight path. In such a scenario, when there is enough time available, ownship should wait and use the traffic probes to verify whether the intruder responds timely and appropriately. Figures 16-18 illustrate how the probes can be used to monitor 3 different responses of traffic to give way. Note how the probes unambiguously indicate the successfulness of the traffic's evasive maneuver. Would traffic not respond (sufficiently) within a certain amount of time, then an evasive maneuver would result, in a way similar to the two scenarios described earlier.

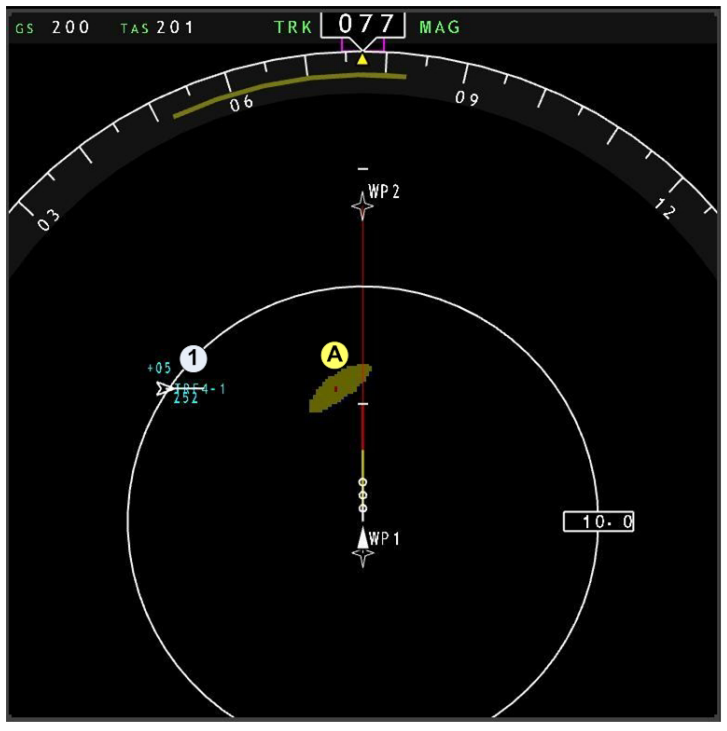

(a)

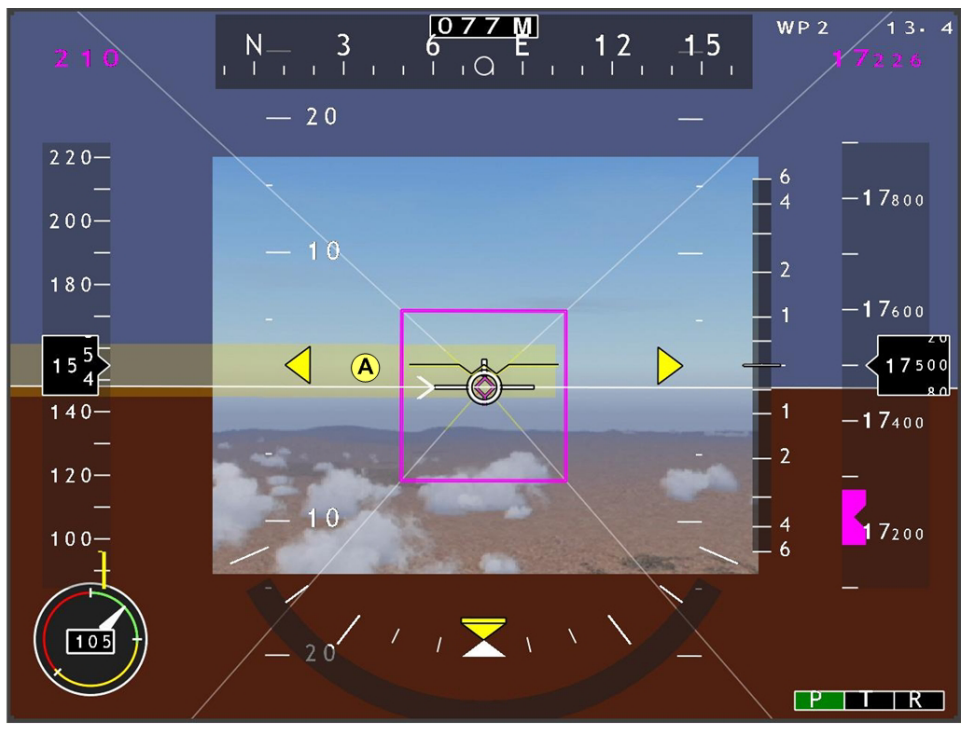

(b)

Figure 15. Initial situation: aircraft 1 coming from the left, causing a future separation conflict.

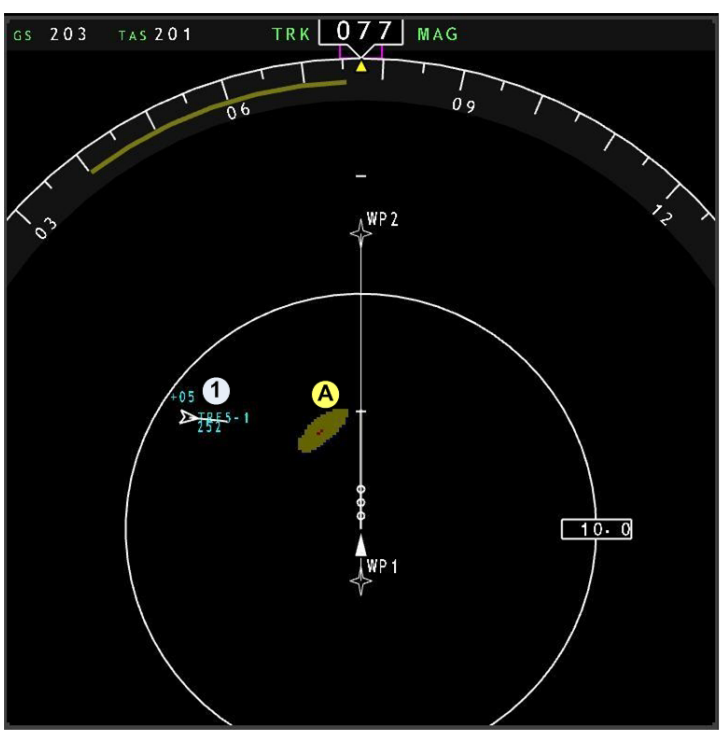

(a)

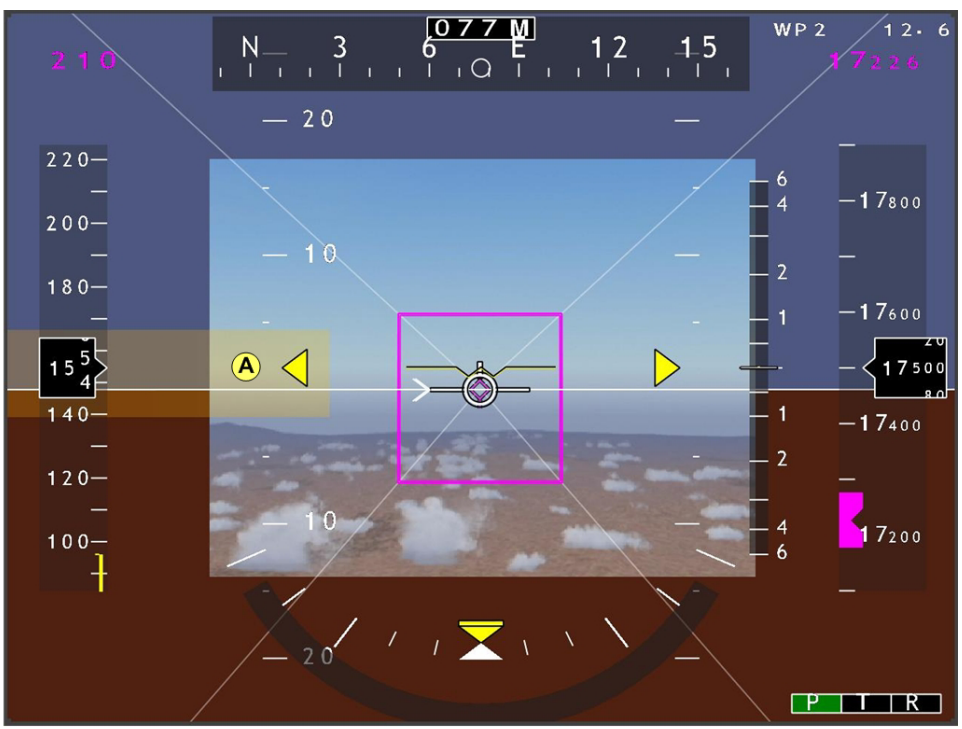

(b)

Figure 16. At T2LOS $=100$ s, aircraft 1 gave way by changing its track 6 deg to the right, causing it to pass behind ownship with $\mathrm{CPA}=1.4 \mathrm{NM}$. 


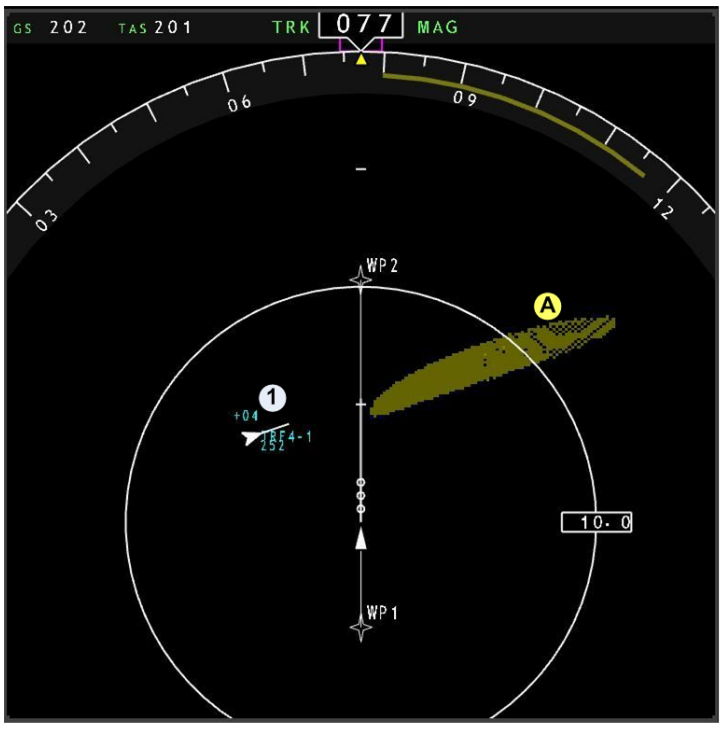

(a)

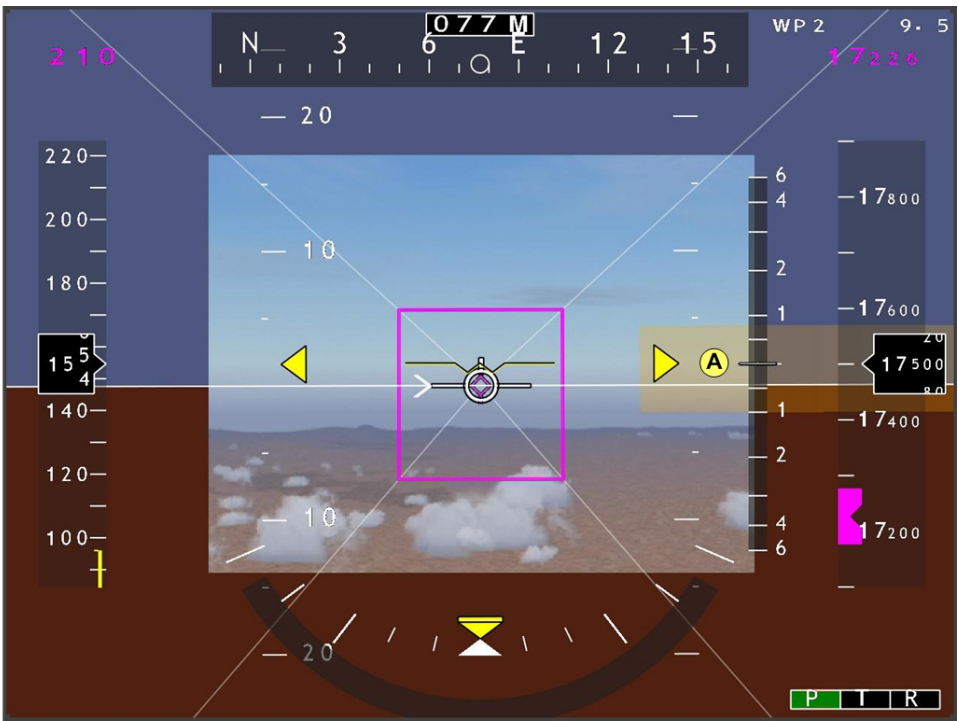

(b)

Figure 17. At T2LOS=100s, aircraft 1 gave way by changing its track 18 deg to the left, causing it to pass in front of ownship with $\mathrm{CPA}=1.2 \mathrm{NM}$.

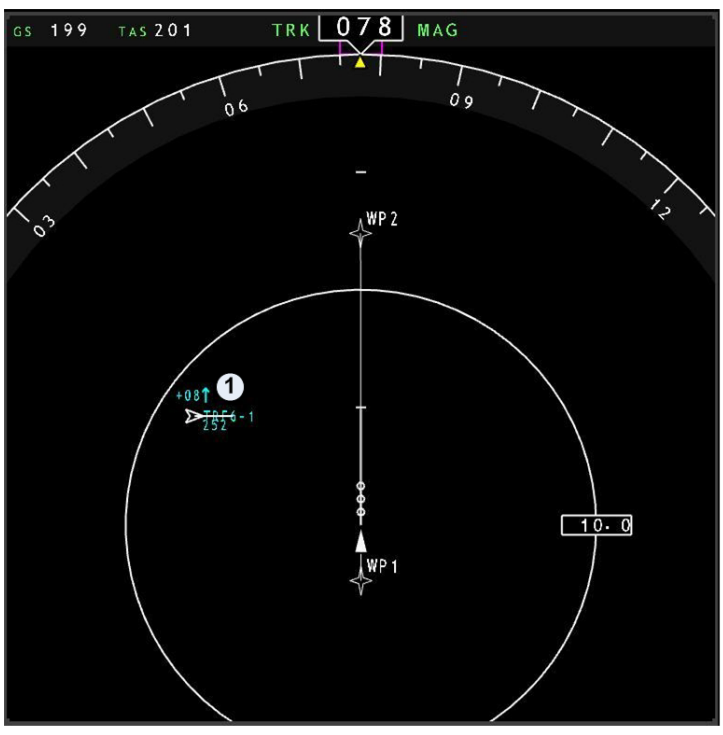

(a)

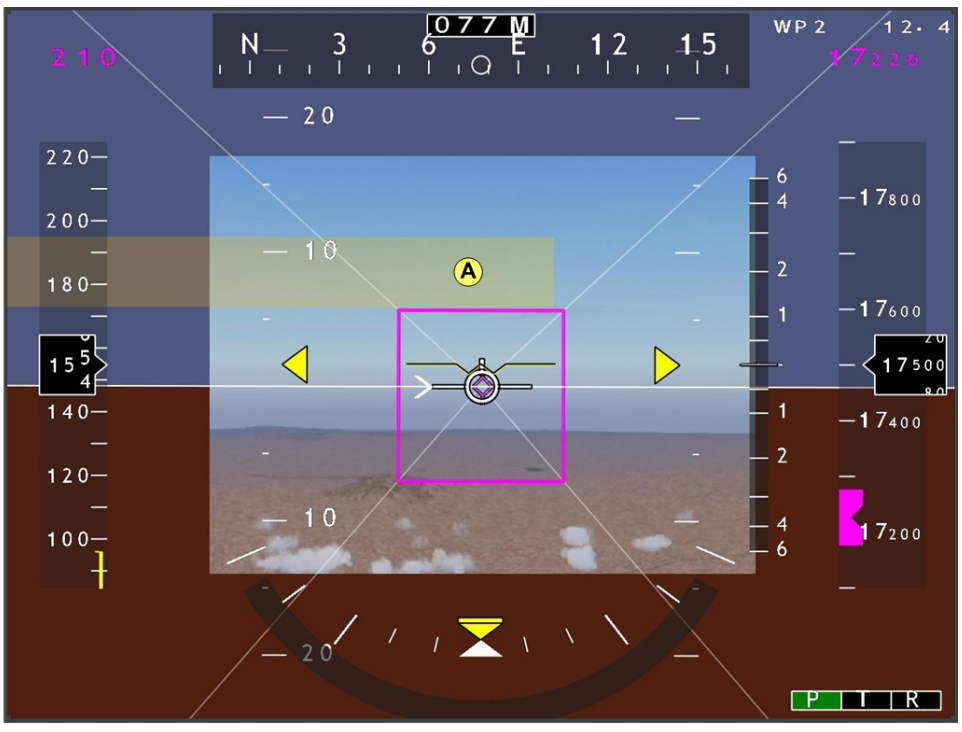

(b)

Figure 18. At T2LOS=100s , aircraft 1 gives way by increasing altitude (climbing $2000 \mathrm{fpm}$ for $30 \mathrm{~s}$ ), causing it to pass over ownship.

\subsection{Solution stability and multivector resolution strategies}

In Ref. [17] we addressed the stability of conflict avoidance options. An avoidance maneuver that is based on the same look-ahead distance as used by the triggered alerting function(s), is predicted to be conflict free, but might not be alert free for up to that distance. Hence, while in the process of executing that maneuver, new alerts, requiring new maneuvers might arise. The design of our conflict avoidance function includes the requirement to be able to control the time-scale on which this is allowed to occur, by setting a pre-defined time for which the conflict alerting function should not be triggered again during the execution of the chosen avoidance maneuver. E.g., to make sure that after its execution, the avoidance maneuver stays valid, without resulting in new conflict alerts for at least a $\Delta \mathrm{T}$ time period, the avoidance maneuver that is selected for execution should be based on a look-ahead horizon of $\mathrm{T}_{\mathrm{LA}}=\mathrm{T}_{\mathrm{AL}}+\Delta \mathrm{T}$. Doing so defines the 
minimum length of single vector resolutions, resulting in a trade-off between solution stability and the possibility to optimally use the available maneuver space.

Figures 19 and 20 demonstrate how scenarios can be used to determine the desired look-ahead time for different concepts of operation. This example scenario consists of three oncoming aircraft, spaced in such a way that it illustrates the trade-off between solution stability and the ability to optimally use the available maneuver space. Figure 20a shows the lateral resolution options on the CDTI, where option 1 resembles to the shortest, and option 3 the largest value for $\Delta \mathrm{T}$. It is shown that large values of $\Delta \mathrm{T}$ might cause undesirably large deviations from the planned path, while less strict solution stability requirements allow for the identification of multivector resolution strategies $(\mathrm{M})$, that stay closer to the planned path and use the available maneuver space more efficiently. Based on the conflict geometry, separation criteria and vehicle maneuverability, multivector solution options can be in identified from the probing data, as indicated by Fig. 20 a.

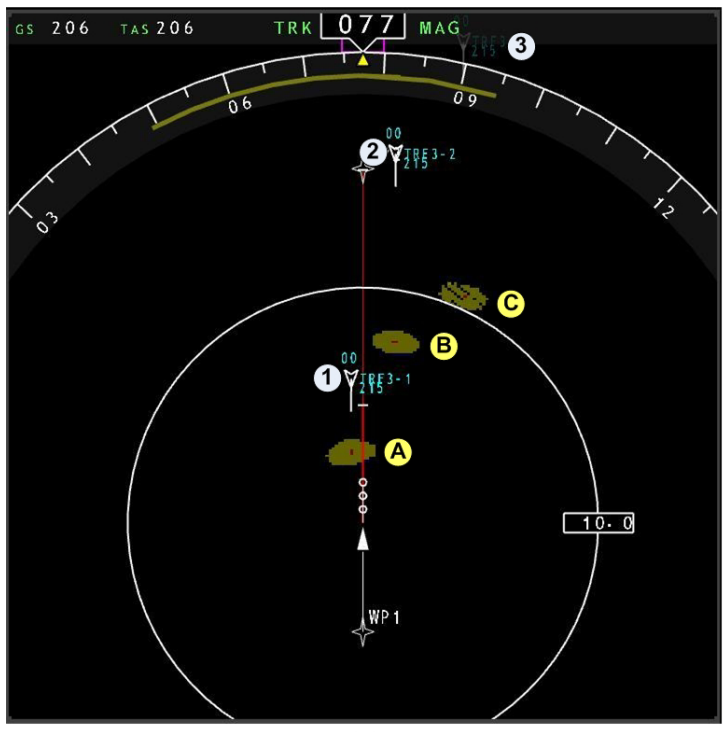

(a)

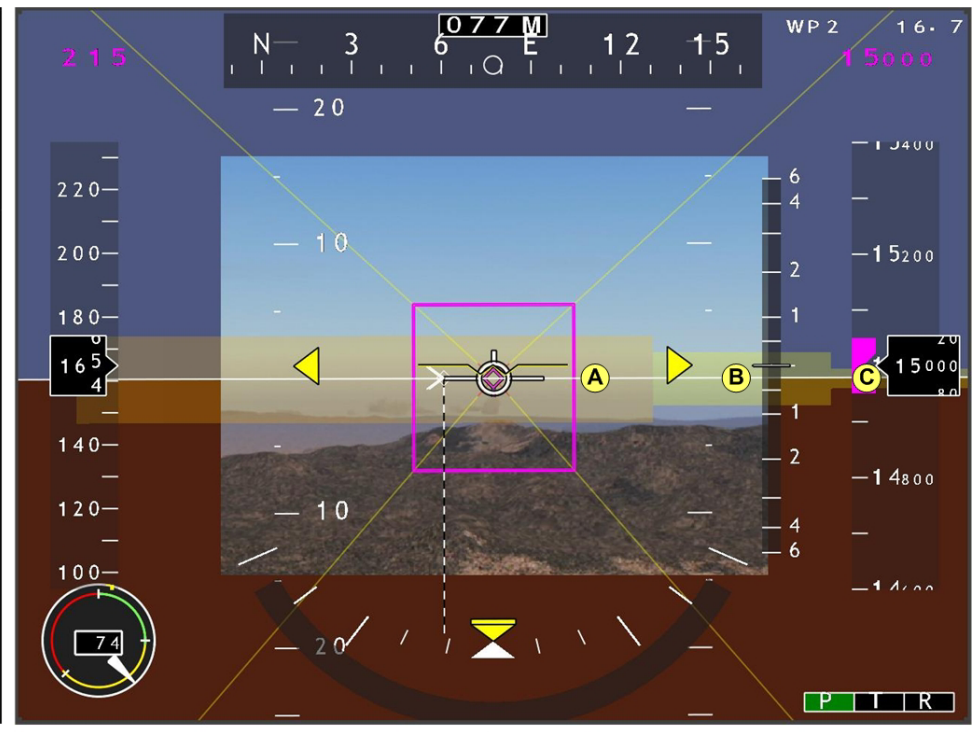

(b)

Figure 19. Example scenario to explore the effect of solution stability requirements.

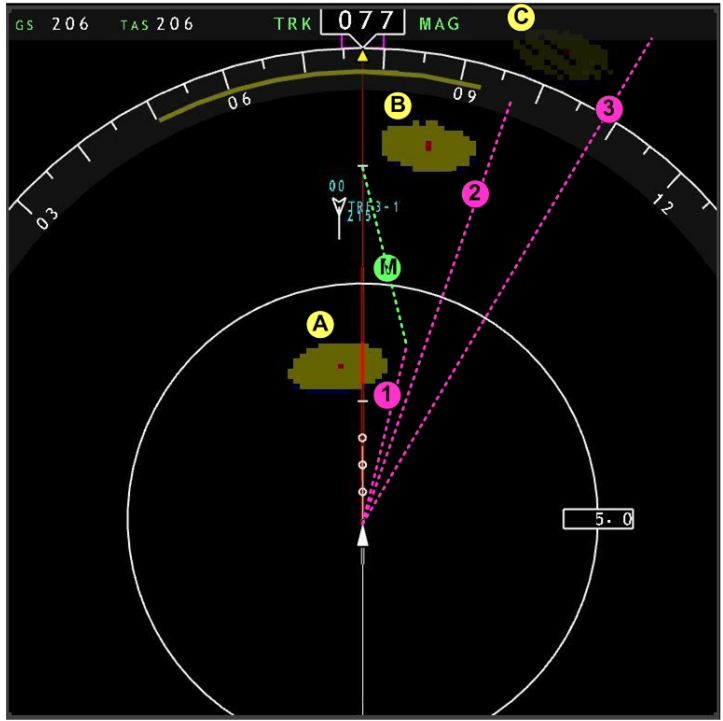

(a)

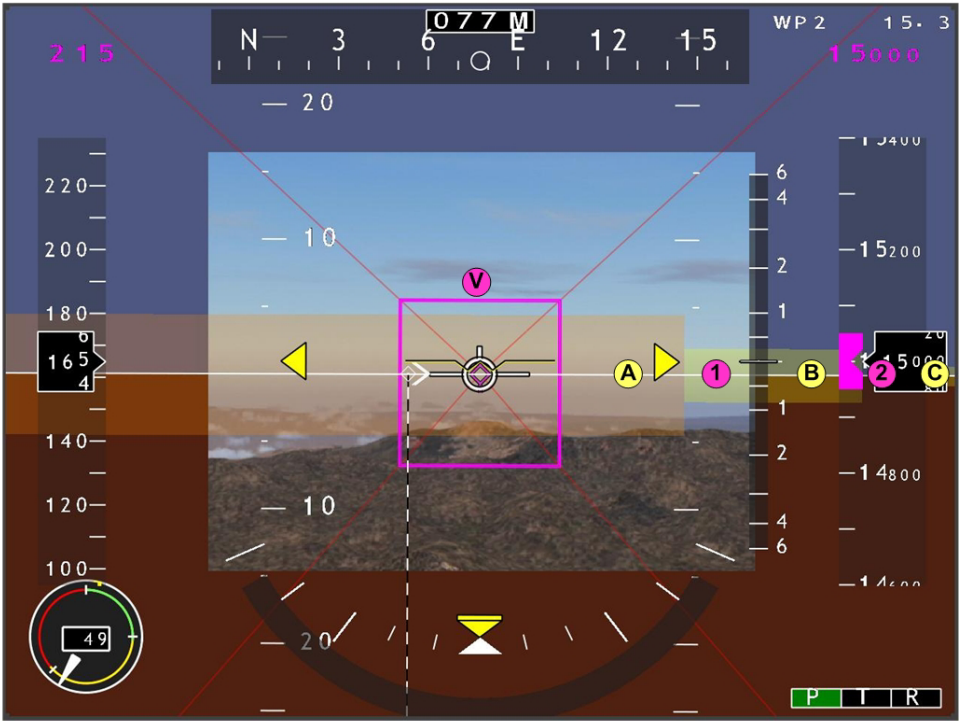

(b)

Figure 20. Existing resolution options, based on different look-ahead times / action horizons 


\section{SUMMARY AND CONCLUSIONS}

SV systems show a computer generated image of the environment with a quality that is independent of actual visibility conditions. The premise is that safety will increase because the timely detection of hazards is no longer impaired by (poor) visibility. Ample research data is available that supports this claim for hazards that are static such as terrain and obstacles. However, for traffic this is not the case. The depiction of other traffic to aid the pilot in detecting a potential future loss of separation has been the subject of a range of studies. These studies implicitly make the assumption that the extrapolation of the current state to the future is performed by the pilot. Additionally, many conflict geometries are possible that will lead to a loss of separation and a collision hazard without the intruding aircraft ever coming within the field of view used to render the synthetic environment of the proposed displays. Research has addressed this issue by designing exocentric views for the depiction of traffic, providing a perspective all-around view relative to ownship.

Rather than using a different viewpoint, the research described in this paper explores the addition of conformally integrated traffic probes into an egocentric SV PFD. The underlying thought is that, although the traffic that is predicted to cause a future loss of separation may not lie within the field of view, the location where the loss of separation is predicted to occur always will. Hence, rather than focusing on the depiction of traffic, which contributes to level 2 SA, the concept pursues spatially integrated depiction of the airspace where a future loss of separation is predicted. This provides readily actionable conflict information, relieving pilots from the traffic position and conflict estimation task and contributing to level 3 SA. Depending upon the concept of operation, the traffic probe can be configured using either a pilot-selectable ANSD or a SST.

The advantages of the concept have been illustrated using several traffic conflict scenarios, including an overtaking scenario involving unmanned aircraft. Additionally, examples were provided illustrating how traffic probes can support pilots in monitoring the conformance of traffic to the priority rules of 14 CFR 91.113. Finally, a scenario addressing solution stability requirements and multivector resolution strategies was provided. With the integration of traffic probes, it has been shown that, independently of traffic depiction, the pilot can immediately see:

- $\quad$ whether the current flight path vector will lead to a loss of separation with traffic;

- where the flight path vector should be kept to be assured that traffic will not become an issue within the chosen time horizons.

\section{REFERENCES}

[1] Ellis, R.S. and M.W. McGreevy, "Perspective Traffic Display Format and Airline Pilot Traffic Avoidance", Human Factors 29(4), 371-382 (1987).

[2] Wickens, C.D. and A.L. Alexander, 3D Navigation and Integrated Hazard Display in Advanced Avionics: Workload, Performance, and Situation Awareness, Technical Report AHFD-04-9/NASA-04-3 (2004).

[3] Xu, X., E.M. Rantanen and C.D. Wickens, Estimation of Conflict Risk Using Cockpit Displays of Traffic Information, Technical Report AHFD-04-11/FAA-04-4 (2004).

[4] Alexander, A.L., C.D. Wickens and D.H. Merwin, "Perspective and Coplanar Cockpit Displays of Traffic Information: Implications for Maneuver Choice, Flight Safety, and Mental Workload", The International Journal of Aviation Psychology, 15(1), 1-21 (2005).

[5] Powell, J.D., C. Jennings and W. Holforty, "Use of ADS-B Perspective Displays to Enhance Airport Capacity", Proceedings of the 24th Digital Avionics Systems Conference, pp. 4D4.1-4D4.9, IEEE/AIAA (2005).

[6] Fiore A.E., R.E. Anderson and L.J. Kapanka, "Historical Approach to Marine Collision Avoidance", Journal of the Institute of Navigation, Vol. 18, No.1 (1971).

[7] Law, J., "TCAS Traffic Display", http://www.theairlinepilots.com/flight/tcastrafficdisplay.htm (2006).

[8] Tadema, J. and E. Theunissen, "An Integrated Conflict Avoidance Concept for Aviation", Proceedings of the 28th Digital Avionics Systems Conference, pp. 5C6.1-5C6.11, Orlando, FL (2009).

[9] Endsley, M.R., "Design and Evaluation for Situation Awareness Enhancements", Proceedings of the Human Factors Society 32nd Annual Meeting, pp.97-101, Santa Monica, CA (1988).

[10] RTCA, Minimum Operational Performance Standards (MOPS) for Aircraft Surveillance Applications System (ASAS), DO-317 (2009).

[11] George, S., FAA UAS Sense and Avoid Workshops April 2009, PowerPoint presentation (2009). 
[12] Tadema, J., E. Theunissen and K.M. Kirk, "Self Separation Support for UAS", Proceedings of the AIAA Infotech@ Aerospace Conference (2010).

[13]FAA, Right-of-way rules: Except water operations, 14CFR91.113 (2001).

[14] Uijt de Haag, M., Bezawada, R., Vana, S., Venable, K., Schnell, T., Cover, M., Haverkamp, D., Etherington, T., Snow, M.P., Theunissen, E. and Rademaker, R.M., Design, Development, Verification and Validation of an Integrated Alerting and Notification Function for an Intelligent Integrated Flight Deck - Phase 1, NASA CR, to be published (2009).

[15] Chamlou, R., "Future Airborne Collision Avoidance - Design Principles, Analysis Plan and Algorithm Development", Proceedings of the 28th Digital Avionics Systems Conference, pp. 6E2.1 - 6E2.17 (2009).

[16] Lambregts, A.A., J. Tadema, R.M. Rademaker and E. Theunissen, "Defining Maximum Safe Maneuvering Authority in 3D Space Required for Autonomous Integrated Conflict Resolution", Proceedings of the 28th Digital Avionics Systems Conference, IEEE/AIAA (2009).

[17] Tadema, J. and E. Theunissen, "Exploring the potential of energy and terrain awareness information in a Synthetic Vision display for UAV control", Proceedings of the SPIE Enhanced and Synthetic Vision 2009, Vol. 7328, pp. 9.1-9.13 (2009).

[18] Theunissen, E., and J. Tadema, "A Simulation Approach for Evaluating Operator-in-the-Loop DSA concepts", Proceedings of AIAA Modeling and Simulation Technologies Conference and Exhibit, AIAA 2008-6347 (2008).

[19] Tadema, J., E. Theunissen, "Design and Evaluation of a GUI for Operator Involvement in Airborne Conflict Detection and Resolution", Proceedings of the 27th Digital Avionics Systems Conference, IEEE/AIAA (2008). 